\title{
AMA0076, a Novel, Locally Acting Rho Kinase Inhibitor, Potently Lowers Intraocular Pressure in New Zealand White Rabbits with Minimal Hyperemia
}

\author{
Sarah Van de Velde, ${ }^{1}$ Tine Van Bergen, ${ }^{1}$ Davine Sijnave,${ }^{1}$ Karolien Hollanders, ${ }^{1}$ \\ Karolien Castermans, ${ }^{2}$ Olivier Defert,${ }^{2}$ Dirk Leysen, ${ }^{2}$ Evelien Vandewalle,,${ }^{1,3}$ Lieve Moons,${ }^{4}$ \\ and Ingeborg Stalmans ${ }^{1,3}$ \\ ${ }^{1}$ The Laboratory of Ophthalmology, KU Leuven, Leuven, Belgium \\ ${ }^{2}$ Amakem Ophthalmics, Diepenbeek, Belgium \\ ${ }^{3}$ Department of Ophthalmology, University Hospitals Leuven (UZ Leuven), Leuven, Belgium \\ ${ }^{4}$ Unit of Animal Physiology and Neurobiology, Biology Department, KU Leuven, Leuven, Belgium
}

Correspondence: Ingeborg Stalmans, University Hospitals Leuven, Department of Ophthalmology, Kapucijnenvoer 33, B-3000, Leuven, Belgium;

Ingeborg.Stalmans@uzleuven.be.

Submitted: August 29, 2013

Accepted: January 9, 2014

Citation: Van de Velde S, Van Bergen T, Sijnave D, et al. AMA0076, a novel, locally acting rho kinase inhibitor, potently lowers intraocular pressure in New Zealand White rabbits with minimal hyperemia. Invest Ophthalmol Vis Sci. 2014;55:1006-1016. DOI:10.1167/iovs.13-13157
Purpose. To determine whether ROCK inhibition for the treatment of glaucoma can be improved by using novel, locally acting Rho kinase (ROCK) inhibitors, such as AMA0076, that lower IOP without inducing hyperemia.

MeTHODS. On-target potency of AMA0076 was compared with other ROCK inhibitors (Y-27632 and Y-39983) and conversion of AMA0076 into its functionally inactive metabolite was evaluated in rabbit eye tissues. Human trabecular meshwork (HTM) cell morphology, actin filaments, and focal adhesion were studied in vitro after exposure to AMA0076. The effect of AMA0076 on IOP was investigated in normotensive rabbits and a new, acute hypertensive rabbit model. Intraocular pressure lowering efficacy of AMA0076 was compared with pharmacologic treatments. Hyperemia after single topical dosing of AMA0076 and Y-39983 was scored.

REsults. AMA0076 and Y-39983 showed similar on-target potency. AMA0076 was most stable in aqueous humor and converted into its metabolite in other eye tissues. Exposure of HTM cells to AMA0076 led to significant and reversible changes in cell shape and a decrease in actin stress fibers and focal adhesions. Both AMA0076 and Y-39983 provided an equivalent IOP control. Compared with latanoprost and bimatoprost, AMA0076 was more potent in preventing the IOP elevation in the acute hypertensive rabbit model. The degree of hyperemia was significantly lower in rabbits treated with AMA0076 then with Y-39983.

ConcLusIONs. AMA0076 is a locally acting ROCK inhibitor that is able to induce altered cellular behavior of HTM cells. Administration of AMA0076 effectively reduces IOP in ocular normotensive and acute hypertensive rabbits without causing distinct hyperemia.

Keywords: ROCK inhibitors, IOP lowering, glaucoma
$T$ The most common form of glaucoma is POAG, which affects more than 2 million persons over 40 years of age in the United States (USA) alone. ${ }^{1}$ Elevated IOP is the only proven, treatable risk factor in multiple forms of glaucoma including POAG. ${ }^{2,3}$ Pharmacologic lowering of IOP has been demonstrated to decrease the risk of progression to POAG in patients with ocular hypertension $(\mathrm{OH})$ and to decrease disease progression in patients with both early and advanced POAG, as well as in those with normal-tension glaucoma. ${ }^{4-9}$

Intraocular pressure is determined by the balance between aqueous humor (AH) production and outflow from the eye. Outflow occurs via two pathways: the trabecular (conventional) and uveoscleral (unconventional) pathways. The main route of outflow is via the conventional pathway. ${ }^{10}$ The primary disease mechanism in POAG involves increased trabecular resistance to $\mathrm{AH}$ outflow via this pathway, ${ }^{11,12}$ resulting in elevated IOP. ${ }^{13}$ Therefore, agents that increase $\mathrm{AH}$ outflow by lowering the trabecular resistance are promising for the treatment of POAG and OH. ${ }^{14}$ Multiple treatment options for IOP control are available, including topically applied and oral drugs, laser treatments, and surgery. ${ }^{15}$ First line topical treatment usually occurs with prostaglandin analogues (PGAs) or beta-blockers, while second line treatment, includes carbonic anhydrase inhibitors, alpha-2 selective antagonists, and cholinergic agents. ${ }^{16}$ Since the declining use of cholinergic agents, such as pilocarpine, it is noteworthy that none of the routinely used medications significantly improves conventional outflow. ${ }^{17}$ As such, a drug that targets this pathway could potentially lead to advancement in the care of $\mathrm{OH}$ and POAG patients. ${ }^{18-20}$ Further, because the conventional pathway is pressure-sensitive $^{18}$ (unlike the unconventional pathway), a drug-targeting conventional outflow may allow for better/ quicker IOP spike recovery compared with drugs that target either $\mathrm{AH}$ production or uveoscleral outflow. ${ }^{21}$

Rho kinase inhibitors constitute a new pharmacologic class of potential drugs that decrease $\mathrm{IOP}^{22-25}$ via effects on the 
trabecular meshwork (TM) and as such increase conventional outflow facility. ${ }^{26}$ Preclinical data indicate that ROCK inhibitors such as Y-27632 (trans-4-[(1R)-1-aminoethyl]-N-4pyridinylcyclohexanecarboxamide dihydrochloride), Y-39983 (4-[(1R)-1-aminoethyl]-N-1H-pyrrolo[2,3-b]pyridin-4-ylbenzamide hydrochloride), and Fasudil (1-(5-isoquinolinylsulfonyl)homopiperazine dihydrochloride) alter the contractility of the TM cells, thereby lowering IOP by facilitating conventional outflow via the relaxation of cells in the TM. ${ }^{22-25,27}$ However, preclinical and clinical studies evaluating ROCK inhibitors have reported mild to severe conjunctival hyperemia, which is most likely due to mechanism-based smooth muscle cell relaxation in conjunctival blood vessels. ${ }^{25,28}$ As a result, most ROCK inhibitors display a narrow therapeutic window. Therefore, Amakem Ophthalmics (Diepenbeek, Belgium) developed a product pipeline of soft ROCK inhibitors. In general, soft drugs, also known as antedrugs or locally acting drugs, are biologically active compounds that are designed to undergo metabolic inactivation by controlled conversion of the parent molecule into a predictable, nontoxic metabolite. ${ }^{29}$ AMA0076 is a novel, potent ROCK inhibitor resulting from Amakem Ophthalmics efforts toward the development of 3-[2-(aminomethyl)-5-[(pyridin-4-yl)carbamoyl] phenyl] benzoates as soft ROCK inhibitors. These compounds contain carboxylic ester moieties, which allow inactivation by esterases and could potentially display an increased therapeutic window. ${ }^{30}$

The goal of the present study was to elucidate the IOPlowering potency and in vivo safety profile of AMA0076. In vitro experiments were performed to investigate the on-target potency and stability of AMA0076, as well as the effect of the ROCK inhibitor on the cytoskeleton and morphology of cultured human TM cells. The IOP-lowering effect was investigated using ocular normotensive rabbits and an acute hypertensive rabbit model. The in vivo effect was compared with Y-39983 and two PGAs, latanoprost and bimatoprost, which are routinely used in the clinic as IOP-lowering agents. Moreover hyperemia was scored after single topical administration and compared with the known ROCK inhibitor, Y39983.

\section{Materials ANd Methods}

\section{In Vitro On-Target Activity Measurements}

The on-target activity of AMA0076 was determined and compared with Y-39983 and Y-27632, using a radiometric protein kinase assay $\left(1 \mu \mathrm{M}\right.$ ATP). ${ }^{31}$ The kinetics of ROCKII inhibition by AMA0076 were characterized by varying the concentrations of ROCKII (aa5-554), peptide substrate (long S6 kinase substrate peptide) and ATP, and measuring inhibition at different time points (up to 120 minutes). Data were analyzed with Michaelis-Menten and Lineweaver-Burk plots.

\section{Animals}

Male New Zealand White (NZW) rabbits (animal facility, KU Leuven, Leuven, Belgium) aged 12 to 14 weeks and weighing 2 to $3 \mathrm{~kg}$, were used in all studies. All experiments were conducted in accordance with the ARVO Statement for the Use of Animals in Ophthalmic and Vision Research and were approved by the ethical committee for the use of animals at $\mathrm{KU}$ Leuven.

\section{Stability in Eye Tissues}

Eye tissues (cornea, conjunctiva, sclera, and AH) were collected from (NZW) rabbits (animal facility, KU Leuven), snap frozen and stored at $-80^{\circ} \mathrm{C}$. On the day of the experiments, tissues were divided into equal pieces and weighted. Next, all samples were immersed into freshly prepared glutathione bicarbonate Ringer's (GBR) buffer oxygenated with $\mathrm{O}_{2} / \mathrm{CO}_{2}(95: 5)$ to $\mathrm{pH} 7.4$, and were spiked with AMA0076 to a final concentration of $20 \mu \mathrm{M}$. After 4-hours incubation at $37^{\circ} \mathrm{C}$ samples were taken at fixed time points and put into ice-cold $\mathrm{ACN} / \mathrm{H}_{2} \mathrm{O}$ (Merck, Darmstadt, Germany) containing the internal standard warfarin (Fluka; Sigma-Aldrich, Steinheim, Germany), for protein precipitation. The remnant of compound was determined by liquid chromatographytandem mass spectrometry (LC/MS-MS) using Analyst Software (AB Sciex, Niewerkerk aan den Ijssel, The Netherlands). The LC/MS-MS apparatus consists of two parts: the LC part (LC20AD; Shimadzu, Duisburg, Germany) and the MS-MS connected to the LC part (3200 Q TRAP LC/MS/MS system; AB Sciex, Applied Biosystems, Niewerkerk aan den Ijssel, The Netherlands). A calibration curve of AMA0076 was made in GBR buffer to calculate the remaining concentration. Based on the data and the weights of the tissues of at least three independent experiments, the rate of metabolism was calculated, assuming first order decay, and specific enzymatic activity was presented as picomole per minute per milligram \pm SEM.

\section{Culture of Human Trabecular Meshwork (HTM) Cells}

Human TM cells were obtained from ScienCell Research Laboratories (Carlsbad, CA) and cultured in poly-L-lysine coated T75 flasks (ScienCell Research Laboratories). Cells were maintained at $37^{\circ} \mathrm{C}$ in a humidified atmosphere of $5 \%$ $\mathrm{CO}_{2}$. Medium was replaced every 2 days and once confluent, cells were passaged in a one-fourth ratio using the trypsinEDTA method. Briefly, cells were incubated with $0.25 \%$ trypsinEDTA (Gibco, Carlsbad, CA) for 5 minutes at $37^{\circ} \mathrm{C}$ and $5 \% \mathrm{CO}_{2}$. Afterwards, cells were centrifuged for 5 minutes at $312 \mathrm{~g}$ and plated. Cells between the third and sixth passage were used in all experiments.

\section{Effect on Human TM Cell Morphology}

Confluent cultures of human TM cells were incubated with AMA0076 $1 \mu \mathrm{M}$ or dimethyl sulfoxide (DMSO) at similar concentration as vehicle. Cultures were observed with an inverted microscope (Primo Vert Zeiss, Oberkochen, Germany) and photographed before application and 60 minutes after administration of AMA0076. The solution was replaced with full medium and incubated again for 15 hours to examine whether the effects of AMA0076 on human TM cells were reversible.

\section{Actin and Vinculin Staining}

Human TM cells were plated on cover glasses in a 12-well plate at a density of $20 \times 10^{3}$ cells per well. When cells reached semi confluence, $1 \mu \mathrm{M}$ of AMA0076 was added and DMSO was used as vehicle. One hour after drug exposure, cells were fixed with $1 \%$ paraformaldehyde (PFA) for 20 minutes at room temperature (RT). F-actin and focal adhesions were stained using the FAK 100 actin cytoskeleton/focal adhesion staining kit (Millipore, Billerica, MA) according to the following protocol. Briefly, cells were permeabilized using $0.1 \%$ Triton X-100 in PBS for 5 minutes at RT and rinsed with PBS. Blocking solution ( $1 \%$ bovine serum albumin; Roche Diagnostics GmbH, Mannheim, Germany) was applied for 30 minutes. The cells were incubated with antivinculin (focal adhesions; 1:300 in blocking solution) at RT for 1 hour and rinsed with PBS. Next, cells were 
incubated at RT for 1 hour with AlexaFluor 488-conjugated goat anti-mouse (1:200) and tetramethylrhodamine-5-(and-6)isothiocyanate (TRITC)-phalloidin (F-actin, 1:100), both diluted in PBS. Cells were washed and mounted with Prolong Gold antifade reagent with $4^{\prime}$, 6-diaminido-2-phenylindole (DAPI; Invitrogen, Carlsbad, CA). Fluorescence was visualized with a confocal laser scanning microscope (FV 1000; Olympus, Melville, NY). To determine whether the effects of AMA0076 on human TM cells were reversible, the drug solution was replaced with full medium and followed by incubation for another 15 hours.

\section{IOP Measurements}

An applanation tonometer (Tono-Pen; Medtronic Solan, Jacksonville, FL) was used to monitor IOP and three recordings per eye were averaged. Before the start of each IOP experiment the OCU-FILM tip cover was renewed and the tonometer was calibrated. The rabbit eyes were anesthetized by topical instillation of $0.4 \%$ oxybuprocaine (Unicaine; Théa Pharma, Schaffhausen, Switzerland) before every IOP measurement.

\section{Ocular Normotensive NZW Rabbits}

In a first set of experiments the IOP-lowering efficacy of single topical administration of AMA0076 at concentrations of $0.1 \%$, $0.3 \%$, and $0.5 \%$ was tested in ocular normotensive NZW rabbits. These results were compared with Y-39983 (0.1\%, $0.3 \%$, and $0.5 \%$ ) and to the PGA latanoprost (Xalatan $0.005 \%$; Pfizer, New York, NY). The contralateral eyes of the AMA0076/Y-39983-treated rabbits received vehicle $\left(\mathrm{H}_{2} \mathrm{O} /\right.$ polyethylene glycol [PEG]). Intraocular pressure measurements were performed immediately before and every hour until 8 hours after single administration of the compounds. Latanoprost was administered at two different time points in two separate experiments. In the first experiment, latanoprost was administered the evening before IOP measurements. In a second experimental set-up, we also tested the effect of latanoprost when dosed in the morning on the day of IOP measurements (as AMA0076 and Y-39983). In both experiments contralateral eyes were treated with saline $(0.9 \% \mathrm{NaCl}$; B. Braun Medical, Bethlehem, PA). In the above experiments all IOP measurements were performed between $8 \mathrm{AM}$ and $5 \mathrm{PM}$.

Furthermore, the effect of AMA0076 0.3\% and latanoprost on IOP was investigated during the night. Compounds were instilled at $8 \mathrm{PM}$ and IOP was measured every 2 hours until 10 hours after administration with a final measurement at 18 hours after instillation. Contralateral eyes of AMA0076 treated animals received vehicle $\left(\mathrm{H}_{2} \mathrm{O} / \mathrm{PEG}\right)$ and saline was administered to the contralateral eyes of rabbits treated with latanoprost.

\section{Ocular Hypertensive Rabbit Model}

Before inducing hypertension, IOP was measured in both eyes. Subsequently, rabbits were anesthetized by an intramuscular injection of $50 \mathrm{mg} / \mathrm{mL}$ ketamine (Ketalar; Pfizer, Ann Arbor, MI) and 2\% Rompun (Bayer Health Care, Pittsburgh, PA). Ocular hypertension was induced by injection of $50 \mu \mathrm{L}$ Viscoat (Alcon Laboratories, Fort Worth, TX) with a blunt ophthalmologic needle (Steriseal ophthalmologic cannula, 27 G); Aspen Medical Europe Ltd., Reddittch, The Netherlands) into the anterior chamber (AC). Before injection, a temporal paracentesis was created with a sharp 26-G needle (Terumo Europe N.V., Leuven, Belgium). After injection, the paracentesis was hydrated with saline to prevent reflux of $\mathrm{AH}$.

\section{Compound Testing in Ocular Hypertensive Model}

In a first experiment, rabbits were divided in different groups, in which the IOP-lowering effect of commercially available IOPlowering drugs, latanoprost and timolol (Timolol 0.5\%; Falcon, Fort Worth, TX) and AMA0076 (0.1\%, 0.3\%, and $0.5 \%)$ was tested. All compounds were administered topically three times at 0 (9 AM), 3 (12 AM) and 6 (3 PM) hours after Viscoat injection. Saline was administered to the contralateral eye of rabbits treated with latanoprost and timolol, whereas vehicle $\left(\mathrm{H}_{2} \mathrm{O} / \mathrm{PEG}\right)$ was used as control in the AMA0076 treated rabbits.

In a second experiment, a head to head comparison between AMA0076 and the PGAs, latanoprost and bimatoprost (Lumigan $0.3 \mathrm{mg} / \mathrm{mL}$; Allergan, Westport, Ireland) was performed. AMA0076 was instilled three times at the day of the experiment. Prostaglandin analogues were administered the evening before induction of hypertension, to have their maximal effect at the time of the IOP elevation challenge.

Intraocular pressure was measured immediately before injection of Viscoat and every hour until 8 hours after inducing $\mathrm{OH}$.

\section{Hyperemia Scoring}

New Zealand White rabbits were used to investigate the hyperemic effect after single dosing of AMA0076, Y-39983, and vehicle. Eyes were photographed using a digital camera (EOS 5D Mark II [100-mm lens]; Canon, Inc., Tokyo, Japan) before, 1, 4, and 8 hours after topical instillation of the compounds. Hyperemia was scored using a scoring system developed by Alcon Research, in which a number is given to the area of vasodilatation: 0 was normal appearance of vessels at limbus and on rectus muscle; 1 was enlargement of vessels normally visible at limbus and on rectus muscle; 2 was branching of vessels at limbus, new vessels are visible; 3 was new vessels visible in open bulbar conjunctival areas; and 4 was diffuse redness in open bulbar conjunctival areas. The numbers 0 and 1 indicate normal scores; 2,3 , and 4 represent adverse events. ${ }^{32}$

\section{Statistical Analysis}

Data at individual time points were analyzed using the Student's $t$-test for independent samples. The time course of IOP changes was analyzed using ANOVA for repeated measurements (GraphPad Prism 5.0; GraphPad Software, Inc., San Diego, CA). $P$ values smaller than 0.05 were considered statistically significant. Data were presented as mean \pm SEM.

\section{RESUlts}

\section{AMA0076 is a Potent, Reversible, and ATP- Competitive ROCK Inhibitor}

Since AMA0076 is a novel ROCK inhibitor, we first determined its on-target potency and compared it with other known ROCK inhibitors (Y-39983 and Y-27632). Analysis showed that AMA0076 is a potent inhibitor of ROCKI and ROCKII with similar potencies on both isoforms (Table 1). AMA0076 was equipotent to Y-39983 and approximately 30-fold more potent than Y-27632 (Table 1). As AMA0076 and Y-39983 showed similar on target potency for ROCKII, we used Y-39983 as a reference ROCK inhibitor for all in vivo studies.

Inhibition of ROCKII by AMA0076 was competitive with respect to ATP and noncompetitive compared with long 56 kinase peptide substrate (Table 2). Inhibition was timeindependent and decreased by dilution of the reaction mixture, suggesting reversible binding (data not shown). 
TABle 1. Relative Potencies ( $\mathrm{IC}_{50}$ Values, $\mathrm{nM}$ ) for ROCK I and II Inhibition by AMA0076 and Reference Compounds

\begin{tabular}{lcc}
\hline Compound & ROCK I & ROCK II \\
\hline AMA0076 & $3.7 \pm 2.0^{*}$ & $2.3 \pm 0.9$ \\
Y-27632 & ND & $54 \pm 23 \dagger$ \\
Y-39983 & ND & $4.3 \pm 2.1^{*}$
\end{tabular}

ND, not determined.

* Average of three independent experiments.

$\dagger$ Average of two independent experiments.

\section{AMA0076 Is Stable in Aqueous Humor and Metabolized in Rabbit Eye Tissues}

AMA0076 differs from other ROCK inhibitors by its soft drug properties. The conversion of AMA0076 into its functionally inactive metabolite was evaluated in NZW rabbit eye tissues (cornea, conjunctiva, and sclera) and AH. The observed degradation rates were converted into specific activities for each tissue, in order to provide a suitable comparison (Table

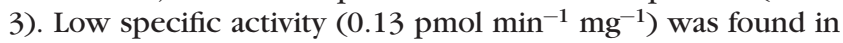
the collected AH samples. Higher specific activities were found in all tested tissues. Cornea displayed the highest specific activity, followed by conjunctiva and sclera (4.81, 1.93, and

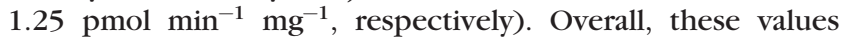
suggest that significant conversion of AMA0076 occurs in vivo in all three tissues, confirming its soft drug properties.

\section{AMA0076 Induces Morphologic and Cytoskeleton Changes in HTM Cells}

Next, the effect of AMA0076 on TM cells was tested in vitro. It has been previously shown that ROCK inhibitors (Y-27632, Y39983, and HA1077) induce morphologic changes in TM cells. $^{22-24,33}$ Therefore, we exposed semiconfluent cultures of human TM cells to AMA0076. Treatment with AMA0076 induced cell-cell separation (retraction) and thinning of the cells after 60 minutes. Replacement of drug solution with full medium resulted in recovery of normal cell morphology (Fig. 1A). To examine whether these morphologic changes were due to cytoskeletal rearrangements, the effect of AMA0076 on the cytoskeleton was investigated by immunofluorescent staining. In control cells, actin filaments assembled into large radial bundles associated with focal adhesions (Fig. 1B, left panel). Exposure to AMA0076 induced distinct alterations in the actin filament organization and focal adhesions of human TM cells (Fig. 1B, middle panel). After treatment with AMA0076, cells lost most of their actin bundles and residual actin filaments became associated with the cell periphery rather than with focal adhesions. Exposure to AMA0076 caused a significant decrease in vinculin expression at the focal adhesion points. All these cytoskeletal changes returned to normal after compound replacement with full medium (Fig. 1B, right panel). There was no effect of the vehicle on

TABLE 2. Kinetic Parameters of In Vitro ROCKII Inhibition by AMA0076

\begin{tabular}{lcc}
\hline & \multicolumn{2}{c}{ Coincubation } \\
\cline { 2 - 3 } & ATP & Peptide Substrate \\
\hline Mode of inhibition & Competitive & Not competitive \\
$\mathrm{K}_{\mathrm{m}}$ & $14 \mu \mathrm{m}$ & $<0.1 \mu \mathrm{m}$ \\
$\mathrm{K}_{\mathrm{i}}$ & $9.4 \mathrm{nM}$ & $\mathrm{ND}$ \\
\hline
\end{tabular}

TABLE 3. Specific Activities (pmol min ${ }^{-1} \mathrm{mg}^{-1}$ ) of NZW Rabbit Eye Tissues and Aqueous Humor for Conversion of AMA0076

\begin{tabular}{lc}
\multicolumn{1}{c}{ Tissue } & Specific Activity \pm SEM \\
\hline Cornea & $4.81 \pm 1.21$ \\
Conjunctiva & $1.93 \pm 0.20$ \\
lera & $1.25 \pm 0.31$ \\
queous humor* & $0.14 \pm 0.01$
\end{tabular}

${ }^{*} 1 \mu \mathrm{L} \mathrm{AH}$ was approximated to $1 \mathrm{mg}$.

general morphology or actin/vinculin staining of HTM cells (data not shown).

Taken together, these data indicate that ROCK inhibition by AMA0076 modifies TM cell morphology.

\section{Single Administration of AMA0076 Effectively Lowers IOP in Normotensive NZW Rabbits}

We then used an in vivo animal model to test the IOP-lowering efficacy of AMA0076. In normotensive NZW rabbits, a single topical administration of the compound significantly decreased IOP compared with control eyes in a dose-dependent manner (Figs. 2A-C). The IOP reduction was significant already 1 hour post dosing, and was sustained for 6 to 8 hours, after which IOP returned to baseline. The peak IOP-lowering efficacy was observed 2 hours post dosing for the $0.5 \%$ and $0.3 \%$ concentrations, and 4 hours post dosing for the $0.1 \%$ concentration. Maximum IOP reduction compared with baseline values after a single dose of AMA0076 at a concentration of $0.5 \%, 0.3 \%$, and $0.1 \%$ was $48 \% \pm 0.32(P=$ $0.03), 39 \% \pm 0.41(P=0.003)$, and $23 \% \pm 0.17(P=0.0006)$, respectively (Fig. 2D). A single administration of Y-39983 also significantly lowered IOP compared with vehicle-treated eyes (Figs. 2E-G). Maximum IOP reduction was reached 2 hours after instillation with Y-39983 at $0.5 \%, 0.3 \%$, and $0.1 \%$ (Fig. $2 \mathrm{H}$ ). Compared with baseline values, IOP was reduced by $38 \% \pm$ $0.55(P=0.01), 34 \% \pm 0.34(P=0.01)$, and $27 \% \pm 0.57(P=$ $0.07)$, respectively. Noteworthy, an attenuated IOP-lowering effect was also observed in the contralateral eye of animals treated with Y-39983. This contralateral effect was not observed in the AMA0076 treated animals. Since this might lead to an underestimation of the IOP reduction by Y-39983, the maximum IOP-lowering effect in all experiments was expressed as compared with baseline.

Finally, we compared the IOP-lowering efficacy of AMA0076 to latanoprost. We tested the effect of latanoprost on IOP after two different time points of administration. First, latanoprost was administered the evening before IOP measurements because, as in humans, latanoprost acts as a prodrug when administered topically to the rabbit eye. Indeed, the cornea serves as a slow release depot and supplies the active form of latanoprost to the anterior segment during an extended period of time. ${ }^{34}$ Next, we also tested the effect of latanoprost when dosed the day of the IOP measurements. In both experiments, administration of latanoprost to ocular normotensive NZW rabbits did not reduce the IOP measured during the day (Figs. 3A, 3B). Compared with contralateral eyes, AMA0076 and latanoprost were able to significantly reduce IOP measured at night, with the peak IOP-lowering effect of both agents being $25.25 \%$ and $22.16 \%$, respectively (Figs. 3C, 3D). In conclusion, the efficacy of AMA0076 was comparable with latanoprost when IOP measurements were performed at night. However, in contrast to latanoprost, AMA0076 reduced IOP also during the day with a potency equal to Y-39983. 

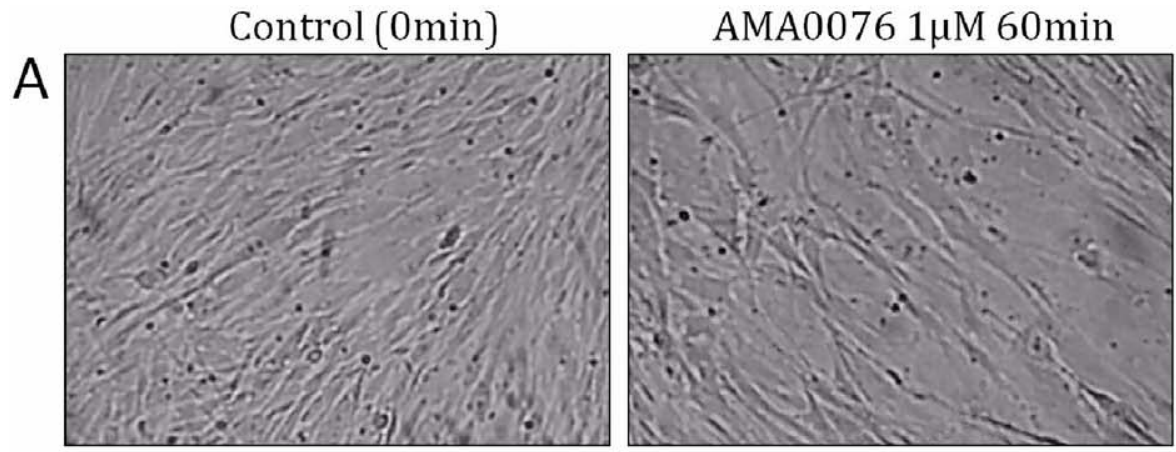

AMA0076 $1 \mu \mathrm{M}$ recovery $15 \mathrm{~h}$
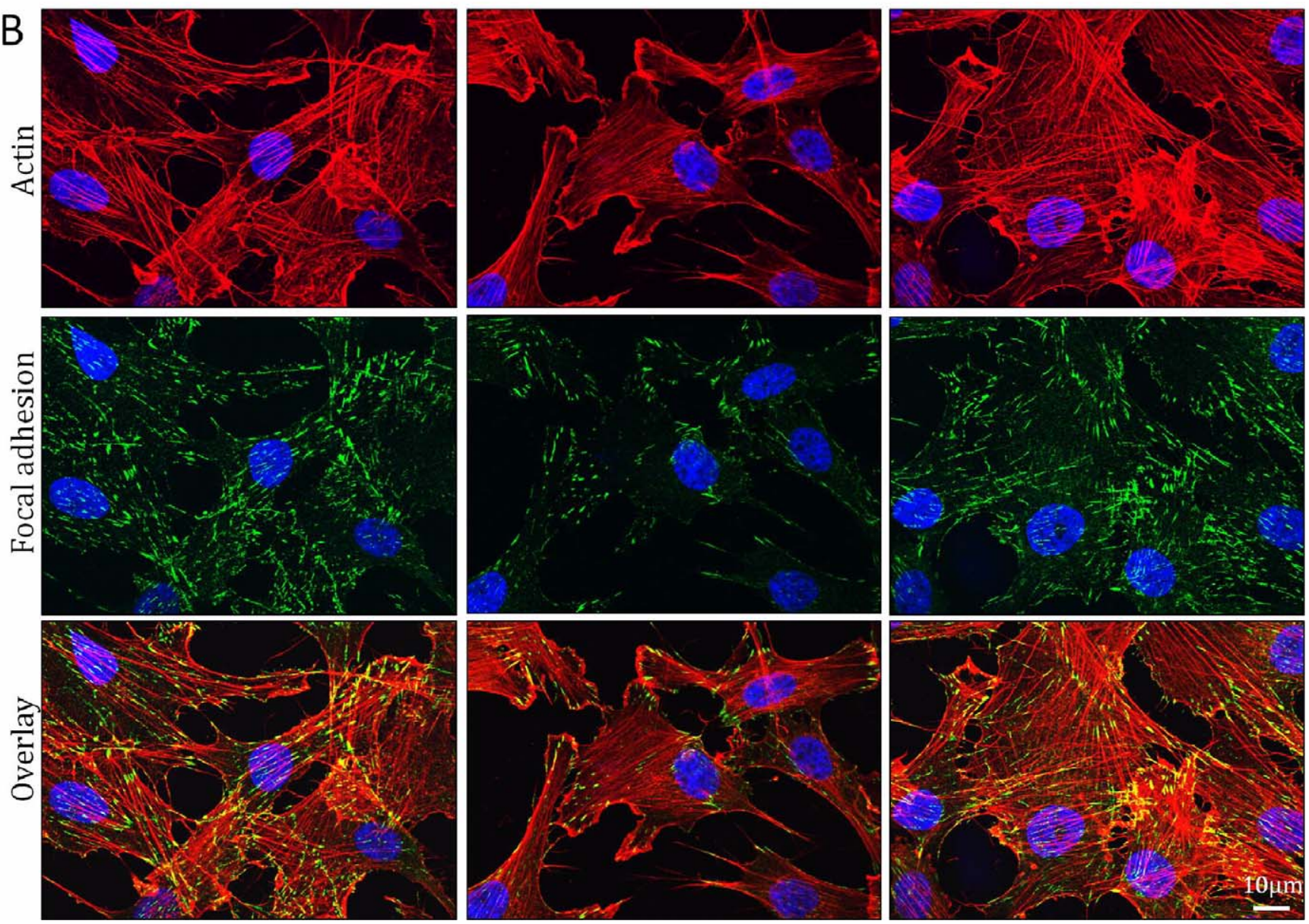

Figure 1. (A) Effect of AMA0076 on morphology of cultured HTM cells. Treatment of HTM cells with $1 \mu \mathrm{m}$ of AMA0076 for 60 minutes resulted in retraction and thinning of the cells. Recovery of normal morphology was observed 15 hours after replacement of drug solutions by full medium. (B) AMA0076 induces changes in actin fibers and focal adhesion (FA) in human TM cells (left panel). Normal distribution of actin fibers (red) and vinculin (green) in human TM cells (middle panel). Distribution of actin and vinculin in human TM cells treated with $1 \mu \mathrm{M}$ AMA0076 for 60 minutes (right panel). Drug solution was removed after and replaced by full medium. Recovery of normal morphology was observed 15 hours later.

\section{AMA0076 Is More Potent Than Prostaglandin Analogues in an Acute Hypertensive Rabbit Model}

To further test the efficacy of AMA0076 against PGAs we set up a hypertensive rabbit model. A single intracameral injection of viscoelastic material (Viscoat) in NZW rabbit eyes resulted in a rapid IOP increase reaching peak pressures of $30 \mathrm{~mm} \mathrm{Hg}$ approximately 5 hours after injection (Fig. 4A). Twenty-four hours after injection of Viscoat, IOP consistently returned to pre-injection levels. Injection of saline did not influence IOP.
In a first experiment, the IOP-lowering agents, latanoprost and timolol, were evaluated in the hypertensive rabbit model. Latanoprost significantly prevented IOP rise after injection with Viscoat compared with saline-treated eyes (Fig. 4B), whereas timolol did not lead to pronounced IOP lowering in this model (Fig. 4C). These data confirm that this hypertensive model is suitable to evaluate compounds that directly target outflow, rather than compounds that decrease $\mathrm{AH}$ production.

Topical administration of AMA0076 significantly and dosedependently prevented the IOP rise compared with vehicle- 

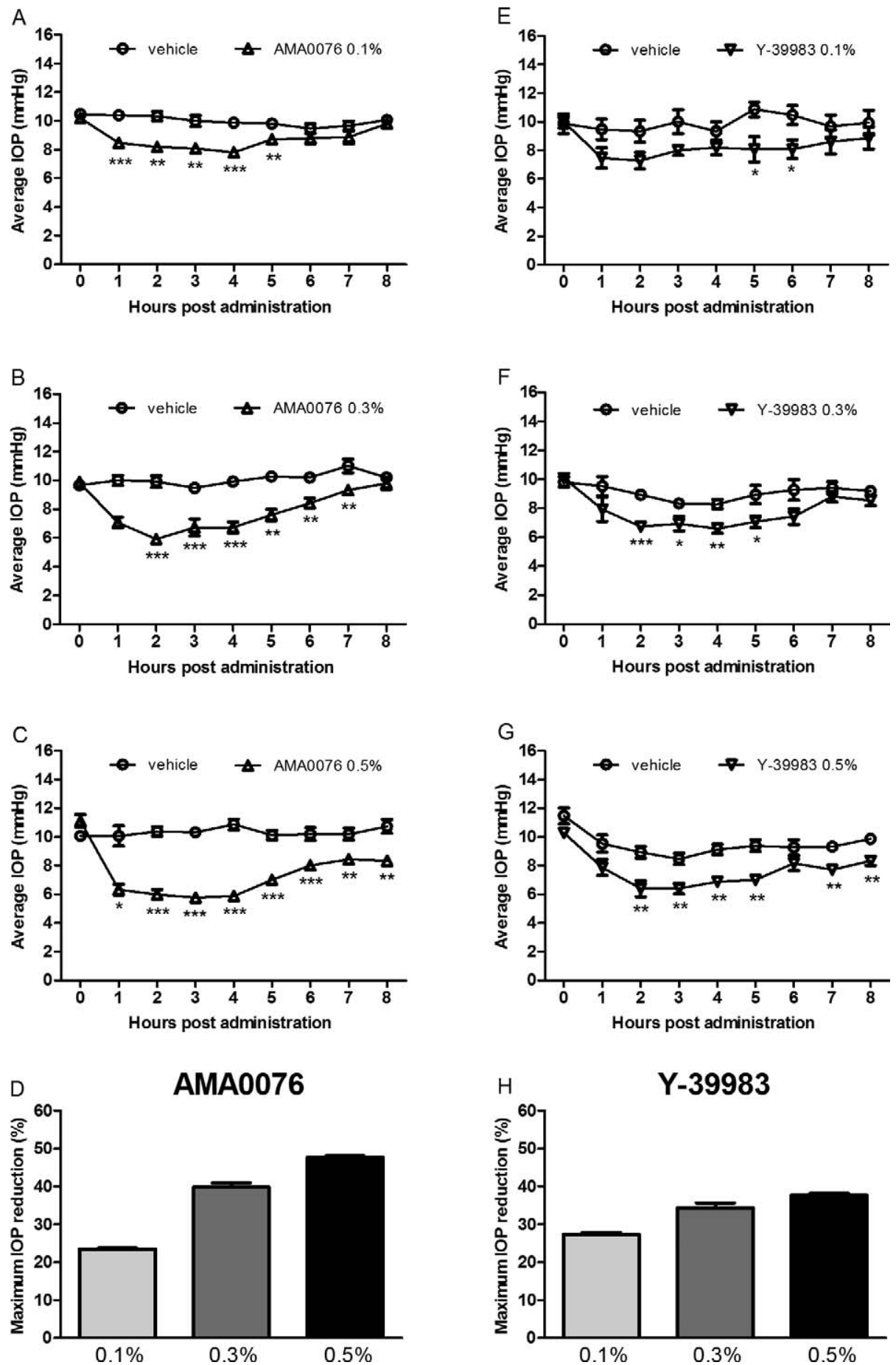

Figure 2. Effect of single topical administration of AMA0076 and Y-39983 on IOP in normotensive NZW rabbits during the day. Contralateral eyes were treated with vehicle $(n=5)$. (A-C) Time course of IOP reduction after topical administration of AMA0076 $(0.1 \%, 0.3 \%$, and $0.5 \%)$ or vehicle $\left({ }^{*} P<0.05,{ }^{* *} P<0.01\right.$, and ${ }^{* * *} P<0.001$ compared with the vehicle group at each time point). (D) Maximum IOP reduction (\%) after topical administration of AMA0076 compared with baseline IOP before administration. (E-G) Time course of IOP reduction after topical administration of Y-39983 or vehicle. ( ${ }^{*} P<0.05,{ }^{* *} P<0.01$, and ${ }^{* * * *} P<0.001$ compared with the vehicle group at each time point). (H) Maximum IOP reduction (\%) after topical administration of Y-39983 compared with baseline IOP before administration. Intraocular pressure measurements were performed between $8 \mathrm{AM}$ and $5 \mathrm{PM}$.

treated eyes (Fig. 4D). AMA0076 0.1\% attenuated the IOP rise induced by the injection of Viscoat compared with the control eye (overall $P<0.0001$; ANOVA), whereas topical administration of AMA0076 $0.3 \%$ and $0.5 \%$ completely inhibited IOP increase (overall $P<0.0001$; ANOVA). Finally, a comparison between AMA0076, latanoprost, and bimatoprost revealed that
AMA0076 was significantly more potent in preventing the IOP elevation in this ocular hypertensive model (overall $P<$ 0.0001; ANOVA; Fig. 5).

Overall, these data show that AMA0076 effectively lowered IOP in an acute hypertensive rabbit model and was more potent than PGAs. 
A

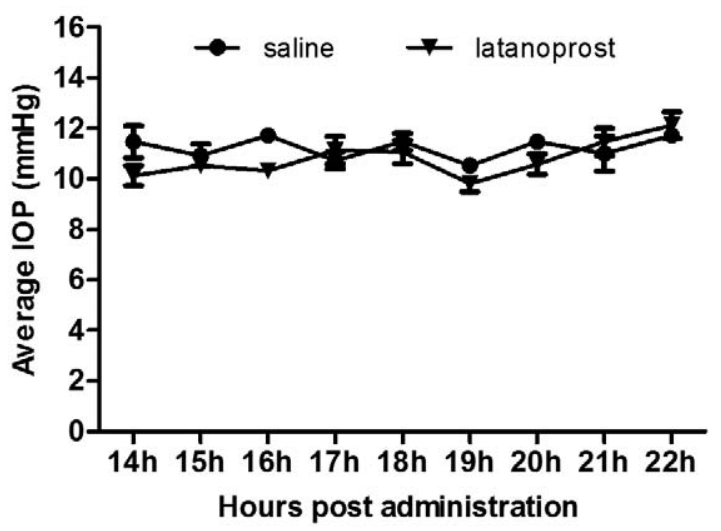

C

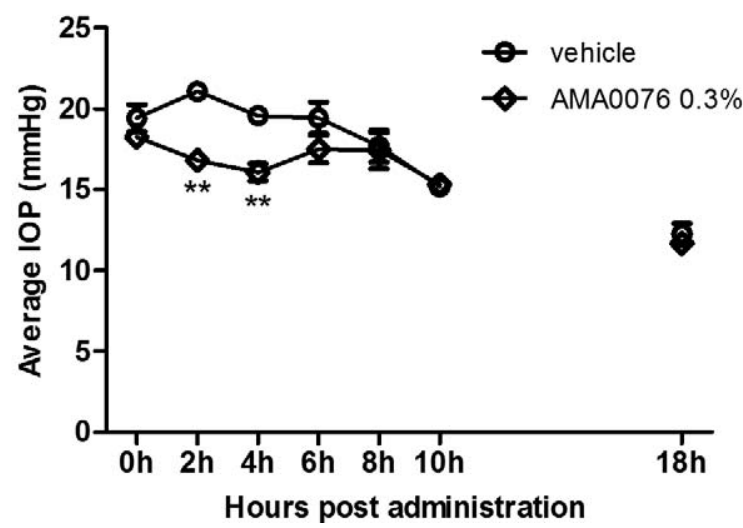

B

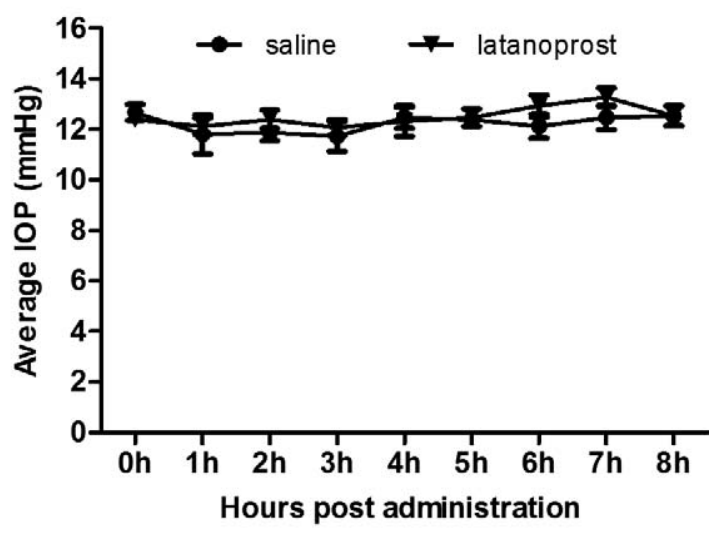

D

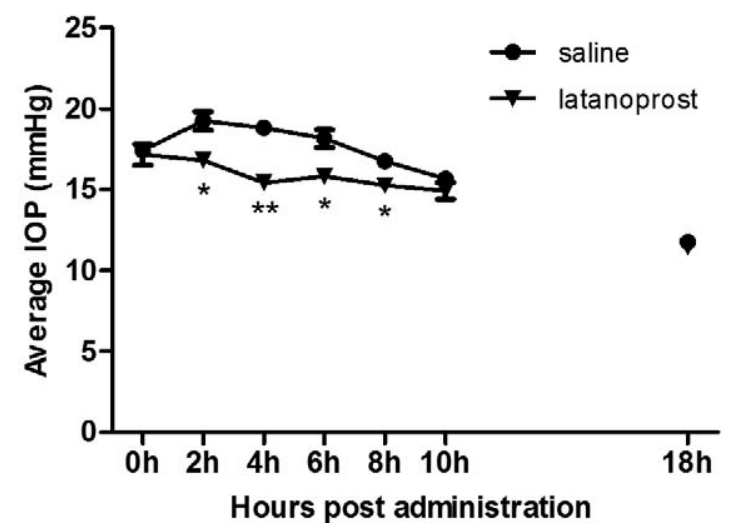

FIGURE 3. Effect of latanoprost on IOP in ocular normotensive NZW rabbits (A) administered the evening before IOP measurements and (B) dosed on the day of the IOP experiment $(n=5)$. Effect of single topical administration of AMA0076 (C) and latanoprost (D) on IOP in ocular normotensive NZW rabbits during the night. Contralateral eyes were treated with vehicle in rabbits treated with AMA0076 or saline for latanoprost $(n=4)$. $(* P<$ $0.05,{ }^{* *} P<0.01$, and ${ }^{* * *} P<0.001$ compared with the vehicle/saline group at each time point).

\section{Minimal Conjunctival Hyperemia With AMA0076}

ROCK inhibitors are known to induce mild to severe transient conjunctival hyperemia. ${ }^{26}$ Therefore the hyperemic effect of AMA0076 0.3\% and Y-39983 0.3\% was investigated in ocular normotensive NZW rabbits. While vehicle-treated eyes did not show distinguishable hyperemic effects, single administration of Y-39983 induced significant hyperemia in the NZW rabbits. In contrast, AMA0076 treatment only caused very mild hyperemia. Table 4 and Figure 6 represent the degree of hyperemia after single topical administration of AMA0076, Y39983 , and vehicle before, 1, 4, and 8 hours after dosing. Thus, compared with Y-39983, AMA0076 induced minimal conjunctival hyperemia in NZW rabbits.

\section{Discussion}

Our results demonstrate that AMA0076, a novel, locally acting ROCK inhibitor significantly reduced IOP after topical administration. In contrast to other ROCK inhibitors, AMA0076 did not cause significant hyperemia at a dose that resulted in strong IOP reduction.

Exposure of human TM cells to AMA0076 led to a decrease in actin bundles and focal adhesions, and thereby induced changes in cell morphology. This finding is in line with the reported effects of other ROCK inhibitors Y-27632, Y-39983, and HA1077. ${ }^{22-24,33}$ Overall, ROCK inhibitors induce cytoskeletal rearrangements and loss of cell-extracellular matrix interactions in TM cells. These modifications might lead to increased intertrabecular pores in vivo and consequently decrease resistance of $\mathrm{AH}$ outflow through the TM, leading to a reduced IOP.

Compared with Y-39983, AMA0076 showed similar on target potencies on ROCKII. The IOP-lowering effect of both ROCK inhibitors was comparable at the tested doses. However, after instillation of Y-39983, an attenuated IOP-lowering effect was also observed in the contralateral vehicle-treated eye. This finding was not observed with AMA0076 and might be explained due to its soft properties. While the efficacy of ROCK inhibitors is promising, they are associated with significant hyperemia due to conjunctival vasodilatation. ${ }^{28} \mathrm{Soft}$ ROCK inhibitors like AMA0076 undergo rapid degradation toward a predefined, functionally inactive metabolite. ${ }^{30}$ Such compounds might reduce side effects and widen the therapeutic window of ROCK inhibitors. ${ }^{35}$ We therefore compared the hyperemic effect of Y-39983, with AMA0076 that is metabolized in rabbit eye tissues. Interestingly, the hyperemic effect of a single topical ocular administration of AMA0076 in NZW rabbits was very mild and significantly lower compared with Y-39983 treatment. The mechanism(s) underlying this phenomenon are yet to be fully elucidated. But it is possible that AMA0076 remaining on the ocular surface is rapidly 
A

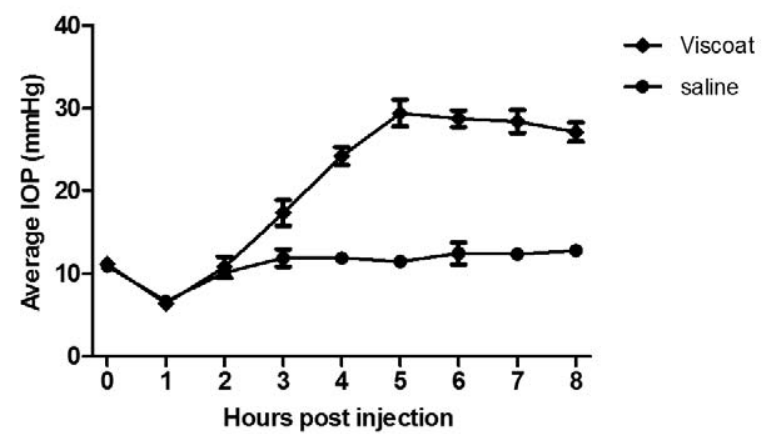

C

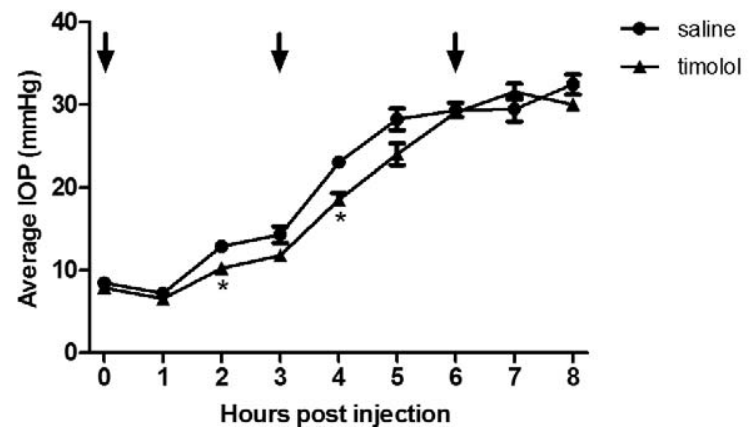

B

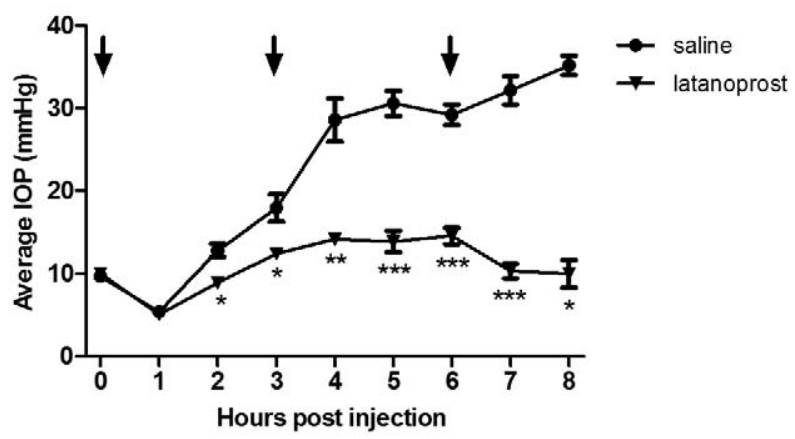

D

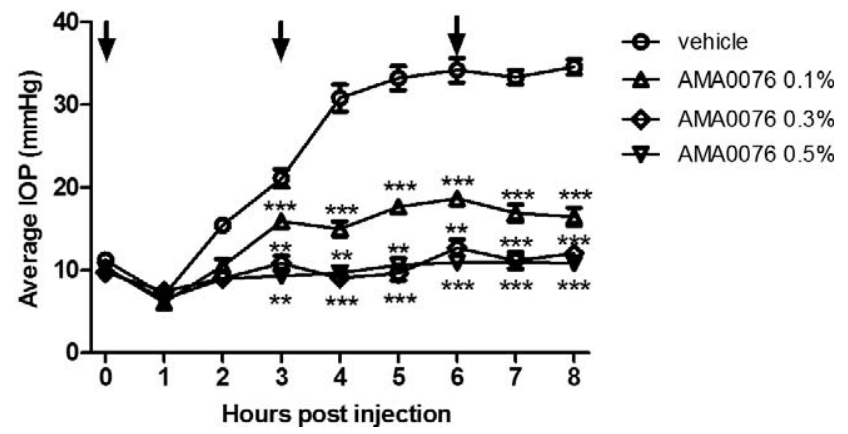

Figure 4. Intraocular pressure lowering effect in hypertensive rabbit model. (A) Effect of Viscoat injection on IOP in NZW rabbits. Control eyes were injected with saline. Intraocular pressure lowering effect of (B) latanoprost and (C) timolol in hypertensive rabbit model. Latanoprost and timolol was topically administered (arrows) to one eye, the contralateral eyes were treated with saline $(n=5)$. (D) Dose response of AMA0076 in hypertensive rabbit model. AMA0076 was topically administered (arrows) to one eye, the contralateral eyes were treated with saline $\left(n=5 ;{ }^{*} P<\right.$ $0.05,{ }^{* *} P<0.01$, and ${ }^{* * *} P<0.001$, compared with the vehicle group at each time point). Intraocular pressure measurements were performed between $8 \mathrm{AM}$ and $5 \mathrm{PM}$.

metabolized and therefore less likely to reach the conjunctival blood vessels to cause hyperemia. However, once AMA0076 gets through the cornea, the remaining is more stable in $\mathrm{AH}$. The relevance of this observation is significant. Several ROCK hyperemia is often suggested to be one of the reasons. ${ }^{28}$ Prevention of hyperemia possibly led to suboptimal dosing with those ROCK inhibitors, resulting in limited IOP lowering. The highest dose of Y-39983 tested in phase 2 trials was $0.1 \%{ }^{36}$ Our results show that maximal IOP lowering is clearly not reached at this dose in NZW rabbits. The ability to dose sufficiently high without causing unacceptable levels of hyperemia is considered to be critical for ROCK inhibitors to be acceptable as IOP-lowering medication for the treatment of glaucoma. $^{28}$

As PGAs are an important treatment modality for glaucoma, we compared the IOP-lowering effect of AMA0076 to PGAs. In our study, latanoprost was unable to reduce IOP in ocular normotensive NZW rabbits during the day. This finding is in line with several previous reports ${ }^{37-39}$ but contradicts with
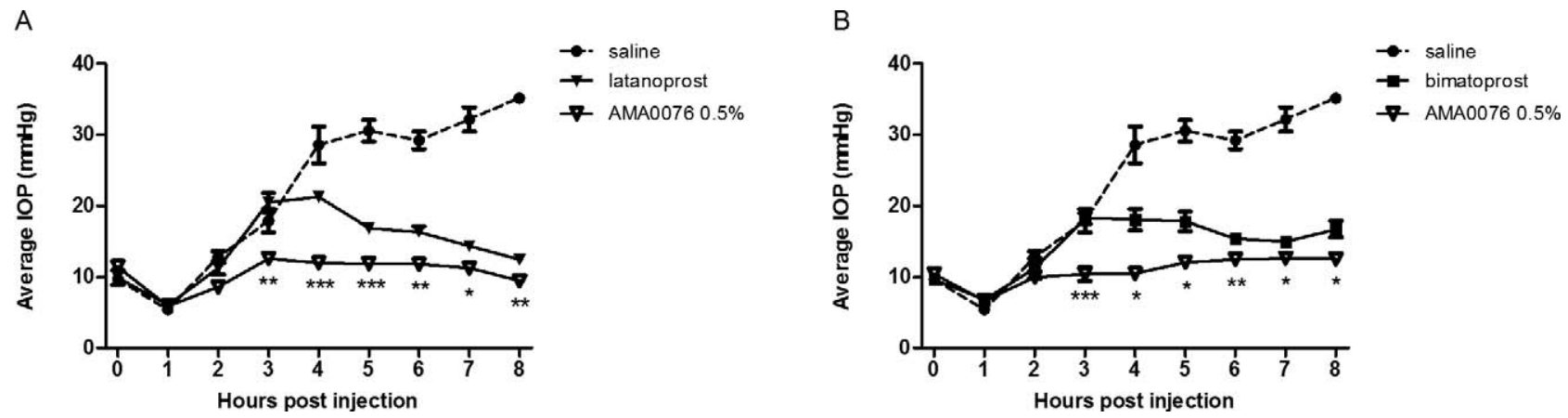

Figure 5. Head to head comparison of AMA0076, latanoprost (A) and bimatoprost (B) in hypertensive rabbit model ( $n=5$ ). AMA0076 was administered three times $(0,3$, and 6 hours after injection of Viscoat), latanoprost and bimatoprost were administered the evening before induction of hypertension $\left(* P<0.05,{ }^{* *} P<0.01\right.$, and ${ }^{* * *} P<0.001$, compared with latanoprost or bimatoprost at each time point). Curve of saline treated eyes (dotted line) was added to each graph as extra information. Intraocular pressure measurements were performed between 8 AM and 5 PM. 
TABLE 4. Scoring of the Hyperemic Effect of AMA0076, Y-39983, and Vehicle After Single Dosing

\begin{tabular}{lclll}
\hline & Before Dosing $(\mathbf{0}$ h) & \multicolumn{1}{c}{$\mathbf{1 ~ h}$} & \multicolumn{1}{c}{$\mathbf{~ h ~}$} & $\mathbf{8 ~ h}$ \\
\hline AMA0076 0.3\% & 0.20 & 0.60 & 0.20 & 0.35 \\
Y-39983 0.3\% & 0.25 & 2.6 & 1.6 & 0.90 \\
$P$ value & 0.69 & 0.00090 & 0.017 & 0.27 \\
Vehicle & 0.33 & 0.33 & 0.38 & 0.33 \\
\hline
\end{tabular}

$n=5 \mathrm{NZW}$ rabbits/compound. Overall $P=0.002$ (AMA0076 versus Y-39983).

others, which did show an IOP-lowering effect of latanoprost in normotensive rabbits. ${ }^{40-42}$ The IOP-lowering effect of latanoprost in rabbits still remains a matter of debate. The reason for the difference may be attributed to variations in method and timing of measurement as well as differences in baseline IOP. ${ }^{40,42}$ This is also reflected by our data. We were only able to reveal an IOP-lowering effect of latanoprost during the night indicating that, in rabbits, the effect of latanoprost depends on the time of day. Rabbits have a clear circadian rhythm of IOP; low during the light phase and high during the dark phase. ${ }^{43,44}$ Furthermore, the observed IOP-lowering effect of latanoprost solely during the time of the day when IOP is highest suggests that the effect on IOP of latanoprost may depend more on baseline IOP than AMA0076. Since IOP fluctuations are known to be a risk factor for glaucoma progression, this finding may be relevant to clinical practice. ${ }^{45}$

In order to further compare the effect of AMA0076 on IOP reduction to currently available glaucoma drugs, a hypertensive rabbit model, based on the injection of viscoelastic agents into the AC, was developed. Viscoelastic agents are commonly used in ocular surgery to maintain AC depth. However, they may cause IOP elevations in the immediate postoperatively period. ${ }^{46}$ In rats the injection of viscoelastics has been used as an $\mathrm{OH}$ model to test the IOP-lowering capacity of several compounds. ${ }^{47}$ Therefore, we adapted the rat model to induce
$\mathrm{OH}$ in rabbits. Using this model, the capacity of compounds to counteract the induced IOP elevation can be investigated. Injection of Viscoat resulted in a rapid and consistent IOP increase in NZW rabbits, most likely a consequence of increased $\mathrm{AH}$ viscosity and disturbed outflow. Several available IOP-lowering drugs, with different mechanisms of action were evaluated in this model. Prostaglandin analogues, which increase mainly uveoscleral $\mathrm{AH}$ outflow, successfully reduced IOP, whereas timolol, which inhibits AH production, did not have an effect. This finding confirms that only compounds which target $\mathrm{AH}$ outflow are able to prevent the IOP rise in this model. This is not unexpected, since AH suppressants presumably further increase AH viscosity in this model, and therefore have no IOP-lowering effect. Overall, our findings show that the induced IOP elevation can be counteracted by outflow facilitating drugs, and therefore this model can be used to test the efficacy of compounds that act via this mechanism. Gupta et al. ${ }^{40}$ also reported the IOP-lowering effect of different IOP-lowering drugs in several hypertensive rabbit models. The IOP-lowering effect of pilocarpine, timolol, and latanoprost was investigated in a water-loading and steroid-induced $\mathrm{OH}$ model. Latanoprost was found to be the most effective in both models. Compared with our model the steroid-induced model is more chronic; however, chronic administration of steroids is associated with a high mortality rate, which was not the case after Viscoat injection. In the water-loading model the IOP increase is very acute and lasts only for 2 hours, whereas in our model a consistent level of $\mathrm{OH}$ was induced for 4 hours.

Our study indicates that AMA0076 was more potent in counteracting the IOP elevation compared with latanoprost and bimatoprost in the hypertensive rabbit model. This might be explained by the different mechanisms of action of the two compound classes. Clinical and animal studies of AH dynamics have reported that PGAs reduce IOP predominantly by increasing uveoscleral outflow and to a lesser extent trabecular outflow facility. ${ }^{48,49}$ Conventional outflow is considered the main route and is believed to be regulated by the cellular behavior and contractility of TM cells. ${ }^{50,51}$ Since ROCK
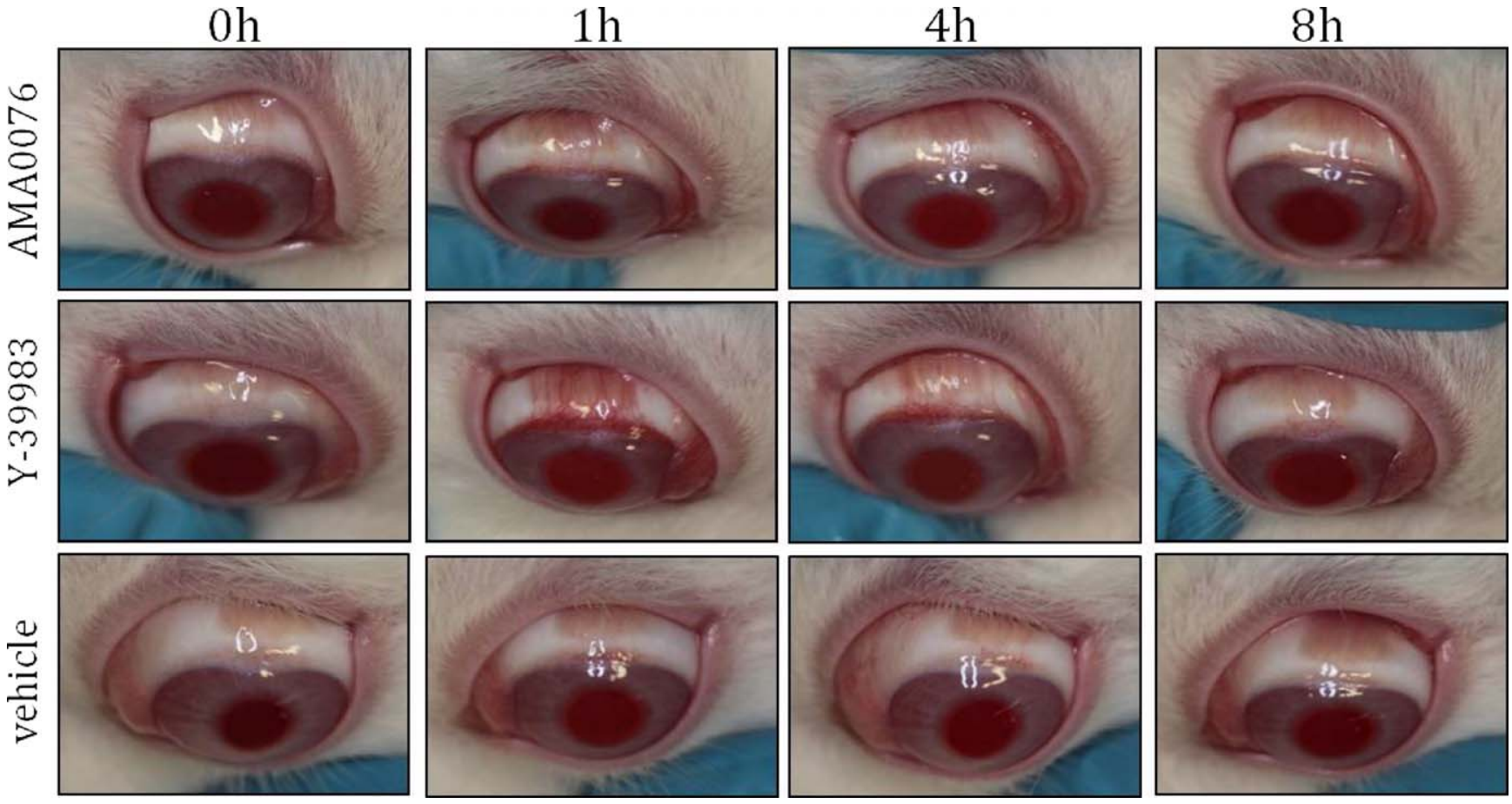

Figure 6. Hyperemic effect of AMA0076, Y-39983, and vehicle in NZW rabbits before, 1, 4, and 8 hours after dosing. 
inhibitors are expected to have a stronger effect on the TM, they have the potential to more effectively lower IOP. ${ }^{24,33}$

In summary, the present study shows that AMA0076 is a potent ROCK inhibitor with a similar IOP-lowering efficacy as Y-39983 and latanoprost, the latter only when dosed at night. Compared with PGAs, AMA0076 was more potent in preventing the IOP elevation in the acute hypertensive model. Due to its soft drug behavior, AMA0076 clearly has an improved tolerability profile with respect to hyperemia, making optimal dosing possible. This increased tolerability window makes AMA0076 a promising new candidate for the treatment of glaucoma.

\section{Acknowledgments}

The authors thank Sofie Beckers, Ann Verbeek, and Martine Leijssen for their technical support and Sandro Boland and Nele Kindt for their contribution in reviewing the draft versions. AMA0076, Y-27632, and Y-39983 were kindly provided by Amakem Ophthalmics.

Supported by the Agentschap voor Innovatie door Wetenschap en Technologie Vlaanderen (IWT), Fonds voor Wetenschappelijk Onderzoek Vlaanderen (FWO), Fund for Research in Ophthalmology (FRO), and Amakem Ophthalmics (Diepenbeek, Belgium).

Disclosure: S. Van de Velde, Amakem Ophthalmics (F); T. Van Bergen, None; D. Sijnave, Amakem Ophthalmics (F); K. Hollanders, Amakem Ophthalmics (F); K. Castermans, Amakem Ophthalmics (I, E); O. Defert, Amakem Ophthalmics (I, E, S), P; D. Leysen, Amakem Ophthalmics (I, E, S), P; E. Vandewalle, None; L. Moons, None; I. Stalmans, Amakem Ophthalmics (F, C)

\section{References}

1. Resnikoff S, Pascolini D, Etya'ale D, et al. Global data on visual impairment in the year 2002. Bull World Health Organ. 2004; 82:844-851.

2. Kwon YH, Fingert JH, Kuehn MH, Alward WL. Primary openangle glaucoma. $N$ Engl J Med. 2009;360:1113-1124.

3. Weinreb RN, Khaw PT. Primary open-angle glaucoma. Lancet. 2004;363:1711-1720.

4. Comparison of glaucomatous progression between untreated patients with normal-tension glaucoma and patients with therapeutically reduced intraocular pressures. Collaborative Normal-Tension Glaucoma Study Group. Am J Ophthalmol. 1998;126:487-497.

5. The Advanced Glaucoma Intervention Study (AGIS): 7. The relationship between control of intraocular pressure and visual field deterioration. Am J Ophthalmol. 2000;130:429440 .

6. Gordon MO, Beiser JA, Brandt JD, et al. The Ocular Hypertension Treatment Study: baseline factors that predict the onset of primary open-angle glaucoma. Arch Ophthalmol. 2002;120:714-720; discussion 829-730.

7. Heijl A, Leske MC, Bengtsson B, Hyman L, Hussein M. Reduction of intraocular pressure and glaucoma progression: results from the Early Manifest Glaucoma Trial. Arch Ophthalmol. 2002;120:1268-1279.

8. Kass MA, Heuer DK, Higginbotham EJ, et al. The Ocular Hypertension Treatment Study: a randomized trial determines that topical ocular hypotensive medication delays or prevents the onset of primary open-angle glaucoma. Arch Ophthalmol. 2002;120:701-713; discussion 829-730.

9. Peeters A, Webers CA, Prins MH, Zeegers MP, Hendrikse F, Schouten JS. Quantifying the effect of intraocular pressure reduction on the occurrence of glaucoma. Acta Ophthalmol. 2010;88:5-11.

10. Toris CB, Yablonski ME, Wang YL, Camras CB. Aqueous humor dynamics in the aging human eye. Am J Ophthalmol. 1999; 127:407-412.
11. Gabelt BT, Kaufman PL. Changes in aqueous humor dynamics with age and glaucoma. Prog Retin Eye Res. 2005;24:612-637.

12. Johnson M. 'What controls aqueous humour outflow resistance?' Exp Eye Res. 2006;82:545-557.

13. Stamer WD, Acott TS. Current understanding of conventional outflow dysfunction in glaucoma. Curr Opin Ophthalmol. 2012;23:135-143.

14. Ferrer E. Trabecular meshwork as a new target for the treatment of glaucoma. Drug News Perspect. 2006;19:151158.

15. Lee DA, Higginbotham EJ. Glaucoma and its treatment: a review. Am J Health Syst Pharm. 2005;62:691-699.

16. Whitson JT. Glaucoma: a review of adjunctive therapy and new management strategies. Expert Opin Pharmacother. 2007;8:3237-3249.

17. Munoz-Negrete FJ, Perez-Lopez M, Won Kim HR, Rebolleda G. New developments in glaucoma medical treatment [in Spanish]. Arch Soc Esp Oftalmol. 2009;84:491-500.

18. Llobet A, Gasull X, Gual A. Understanding trabecular meshwork physiology: a key to the control of intraocular pressure? News Physiol Sci. 2003;18:205-209.

19. Stumpff F, Wiederholt M. Regulation of trabecular meshwork contractility. Ophthalmologica. 2000;214:33-53.

20. Wiederholt M. Direct involvement of trabecular meshwork in the regulation of aqueous humor outflow. Curr Opin Ophthalmol. 1998;9:46-49.

21. Brubaker RF. Targeting outflow facility in glaucoma management. Surv Opbthalmol. 2003;48(suppl 1);S17-S20.

22. Honjo M, Inatani M, Kido N, et al. Effects of protein kinase inhibitor, HA1077, on intraocular pressure and outflow facility in rabbit eyes. Arch Ophthalmol. 2001;119:1171-1178.

23. Honjo $\mathrm{M}$, Tanihara $\mathrm{H}$, Inatani $\mathrm{M}$, et al. Effects of rho-associated protein kinase inhibitor Y-27632 on intraocular pressure and outflow facility. Invest Ophthalmol Vis Sci. 2001;42:137-144.

24. Rao PV, Deng PF, Kumar J, Epstein DL. Modulation of aqueous humor outflow facility by the Rho kinase-specific inhibitor Y27632. Invest Ophthalmol Vis Sci. 2001;42:1029-1037.

25. Tokushige $H$, Inatani $M$, Nemoto $S$, et al. Effects of topical administration of $y-39983$, a selective rho-associated protein kinase inhibitor, on ocular tissues in rabbits and monkeys. Invest Ophthalmol Vis Sci. 2007;48:3216-3222.

26. Colligris B, Crooke A, Huete F, Pintor J. Potential role of Rhoassociated protein kinase inhibitors for glaucoma treatment. Recent Pat Endocr Metab Immune Drug Discov. 2012;6:8998.

27. Rao VP, Epstein DL. Rho GTPase/Rho kinase inhibition as a novel target for the treatment of glaucoma. BioDrugs. 2007; 21:167-177.

28. Mandell KJ, Kudelka RM, Wirostko B. Rho kinase inhibitors for treatment of glaucoma. Exp Rev Ophthalmol. 2011;6:611622.

29. Bodor N, Buchwald P. Retrometabolic drug design: principles and recent developments. Pure Appl Chem. 2008;80:16691682.

30. Boland S, Defert O, Alen J, et al. 3-[2-(Aminomethyl)-5[(pyridin-4-yl)carbamoyl]phenyl] benzoates as soft ROCK inhibitors. Bioorg Med Chem Lett. 2013;23:6442-6446.

31. Anastassiadis T, Deacon SW, Devarajan K, Ma H, Peterson JR. Comprehensive assay of kinase catalytic activity reveals features of kinase inhibitor selectivity. Nat Biotechnol. 2011; 29:1039-1045.

32. Ogundele AB, Earnest $\mathrm{D}$, McLaughlin MA. In vivo comparative study of ocular vasodilation, a relative indicator of hyperemia, in guinea pigs following treatment with bimatoprost ophthalmic solutions $0.01 \%$ and $0.03 \%$. Clin Opbthalmol. 2010;4: 649-652. 
33. Ramachandran C, Patil RV, Combrink K, Sharif NA, Srinivas SP. Rho-Rho kinase pathway in the actomyosin contraction and cell-matrix adhesion in immortalized human trabecular meshwork cells. Mol Vis. 2011;17:1877-1890.

34. Sjoquist B, Basu S, Byding P, Bergh K, Stjernschantz J. The pharmacokinetics of a new antiglaucoma drug, latanoprost, in the rabbit. Drug Metab Dispos. 1998;26:745-754.

35. Bodor N, Buchwald P. Soft drug design: general principles and recent applications. Med Res Rev. 2000;20:58-101.

36. Tanihara $\mathrm{H}$, Inatani $\mathrm{M}$, Honjo $\mathrm{M}$, Tokushige $\mathrm{H}$, Azuma J, Araie M. Intraocular pressure-lowering effects and safety of topical administration of a selective ROCK inhibitor, SNJ-1656, in healthy volunteers. Arch Ophthalmol. 2008;126:309-315.

37. Dinslage S, McLaren J, Brubaker R. Intraocular pressure in rabbits by telemetry II: effects of animal handling and drugs. Invest Ophthalmol Vis Sci. 1998;39:2485-2489.

38. Ishii K, Tomidokoro A, Nagahara M, et al. Effects of topical latanoprost on optic nerve head circulation in rabbits, monkeys, and humans. Invest Ophthalmol Vis Sci. 2001;42: 2957-2963.

39. Orihashi M, Shima Y, Tsuneki H, Kimura I. Potent reduction of intraocular pressure by nipradilol plus latanoprost in ocular hypertensive rabbits. Biol Pharm Bull. 2005;28:65-68.

40. Gupta SK, Agarwal R, Galpalli ND, Srivastava S, Agrawal SS, Saxena R. Comparative efficacy of pilocarpine, timolol and latanoprost in experimental models of glaucoma. Methods Find Exp Clin Pharmacol. 2007;29:665-671.

41. Pintor J, Pelaez T, Peral A. Adenosine tetraphosphate, Ap4, a physiological regulator of intraocular pressure in normotensive rabbit eyes. J Pharmacol Exp Ther. 2004;308:468-473.
42. Sarchahi AAG, Toghraie, FS. Effects of latanoprost and pilocarpine combination on the intraocular pressure and pupil size of normal rabbits. Iran J Vet Res. 2011;12:298-303.

43. Gregory DS. Timolol reduces IOP in normal NZW rabbits during the dark only. Invest Ophthalmol Vis Sci. 1990;31:715721.

44. Lee TC, Kiuchi Y, Gregory DS. Light exposure decreases IOP in rabbits during the night. Curr Eye Res. 1995;14:443-448.

45. Asrani S, Zeimer R, Wilensky J, Gieser D, Vitale S, Lindenmuth $\mathrm{K}$. Large diurnal fluctuations in intraocular pressure are an independent risk factor in patients with glaucoma. J Glaucoma. 2000;9:134-142.

46. Higashide $T$, Sugiyama $K$. Use of viscoelastic substance in ophthalmic surgery - focus on sodium hyaluronate. Clin Ophthalmol. 2008;2:21-30.

47. Benozzi J, Nahum LP, Campanelli JL, Rosenstein RE. Effect of hyaluronic acid on intraocular pressure in rats. Invest Ophthalmol Vis Sci. 2002;43:2196-2200.

48. Toris CB, Camras CB, Yablonski ME, Brubaker RF. Effects of exogenous prostaglandins on aqueous humor dynamics and blood-aqueous barrier function. Surv Ophthalmol. 1997; 41(suppl 2):S69-S75.

49. Weinreb RN, Toris CB, Gabelt BT, Lindsey JD, Kaufman PL. Effects of prostaglandins on the aqueous humor outflow pathways. Surv Ophthalmol. 2002;47(suppl 1):S53-S64.

50. Fautsch MP, Johnson DH. Aqueous humor outflow: what do we know? Where will it lead us? Invest Ophthalmol Vis Sci. 2006; $47: 4181-4187$.

51. Zhang M, Maddala R, Rao PV. Novel molecular insights into RhoA GTPase-induced resistance to aqueous humor outflow through the trabecular meshwork. Am J Physiol Cell Physiol. 2008;295:C1057-C1070. 